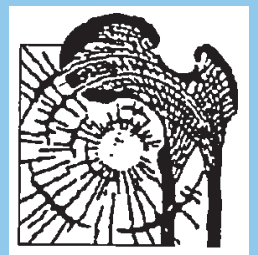

\title{
Informationen der Gesellschaft
}

\section{Vorstand}

\section{Präsident}

Herr Prof. Dr. Hans-Christof Schober

Klinikum Südstadt Rostock

Südring 81

18059 Rostock

Tel: (+ 49) 0381-4401-5000

Fax: (+49) 0381-4401-5099

E-Mail: Hans-christof.schober@kliniksuedrostock.de

\section{Vizepräsidentin}

Frau Prof. Dr. Heide Siggelkow Universitätsmedizin Göttingen (UMG)

Georg-August-Universität

Robert-Koch-Str. 40

37075 Göttingen

Tel.: (+49) 551-63374622

Fax: (+49) 0551-53374646

E-Mail:

heide.siggelkow@endokrinologikum.com

\section{Schriftführerin}

Priv.-Doz. Dr. med. Gabriele Lehmann

Klinik Innere Medizin III

Am Klinikum 1

07747 Jena

Tel.: + 49 (0) 3641/93243-27

Fax: + 49 (0) 3641/93 260-12

E-Mail: gabriele.lehmann@med.uni-jena.de

\section{DGO im Internet}

www.dgosteo.de

\section{Geschäftsstelle}

\section{bei der Schriftführerin}

Priv.-Doz. Dr. med. Gabriele Lehmann

Klinik Innere Medizin III

Am Klinikum 1

07747 Jena

Tel.: + 49 (0) 3641/93 243-27

Fax: + 49 (0) 3641/93 260-12

E-Mail: gabriele.lehmann@med.uni-jena.de

\section{Mitgliedsbeitrag}

Der Mitgliedsbeitrag 2019 beträgt für ordentliche Mitglieder Euro 120,-.

Der Mitgliedsbeitrag 2019 beträgt für studentische Mitglieder Euro 10,--

Der Beitrag schließt den Bezug der Fachzeitschrift Osteologie ein.

\section{Beitragskonto}

DGO e. V.

Sparkasse Göttingen

BLZ: 260500 01, Kontonummer: 56017163

IBAN: DE07 260500010056017163

BIC (SWIFT): NOLADE21GOE
Arbeitskreise

\section{AK Diabetes und Knochen}

Sprecher:

Prof. Dr. med. Lorenz Hofbauer

Universitätsklinikum Dresden

Medizinische Klinik und Poliklinik III

Bereich Endokrinologie,

Diabetes \& Osteologie

Fetscherstr. 74

01307 Dresden

Tel.: + 49(0) 351/458-3173

Fax: + 49(0) 351/458-4309

E-Mail:

lorenz.hofbauer@uniklinikum-dresden.de

und

Prof. Dr. med. Christian Meier

Facharzt FMH für Endokrinologie-Diabeto-

logie u. Innere Medizin

Universitätsspital Basel

Missionsstr. 24

4055 Basel, Schweiz

Tel: + 41 (0) 61/2649797

Fax: + 41 (0) 61/2649796

E-Mail: christian.meier@unibas.ch

\section{AK Osteotechnologie}

Sprecher:

Univ.-Prof. Dr. rer. nat. Kay Raum Charité - Universitätsmedizin Berlin Julius Wolff Institute \& Berlin-Brandenburg School for Regenerative Therapies

Augustenburger Platz 1

13353 Berlin

Tel.: + 49(0) 30/450-539503

Fax: + 49(0) 30/450-539952

E-Mail: kay.raum@charite.de

IMPRESSUM

Verantwortlich für den Inhalt

„DGO - Informationen der

Gesellschaft"

Priv.-Doz. Dr. Gabriele Lehmann, Jena 